\title{
Schlimm, schlimmer, am schlimmsten oder eine Runde Katastrophenquartett?
}

\author{
Elisabeth Meyer
}
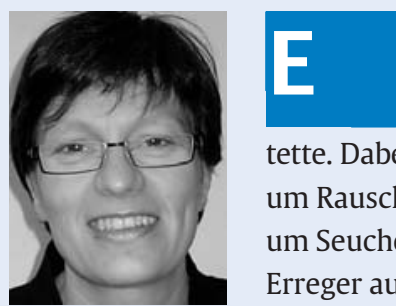

s gibt Tierquartette, Auto- oder Fußballquartette. Und es gibt als makabreren Zeitvertreib sogenannte Katastrophenquartette. Dabei geht es um Tyrannen und Völkermörder, um Rauschgift, Ungeziefer, Atomkraftwerke oder eben um Seuchen [1]: „Die tödlichsten Krankheiten und ihre Erreger auf je 32 Spielkarten“.

Elisabeth Meyer
Der Spiegel kommentiert diese Quartette unter der Überschrift Böses Spielchen [2]: „Daten vergleichen ist eine der einfachsten Formen des Kartenspiels: Zwei Spieler treten mit den Werten ihrer Karten in Wettstreit, der mit den höheren Punktzahl gewinnt. Vor allem kleine Jungs lieben dieses Prinzip, lieben Quartettspiele. [...] Quartette sind Kinderkram? Nicht unbedingt.“

Stimmt! Und wie eine solche Runde Katastrophenquartett mutet die Diskussion um resistente Erreger an. Britische Wissenschaftler haben im Auftrag der Regierung Untersuchungen zu den stetig ansteigenden Antiinfektiva-Resistenzen vorgenommen [3]. Die Ergebnis-

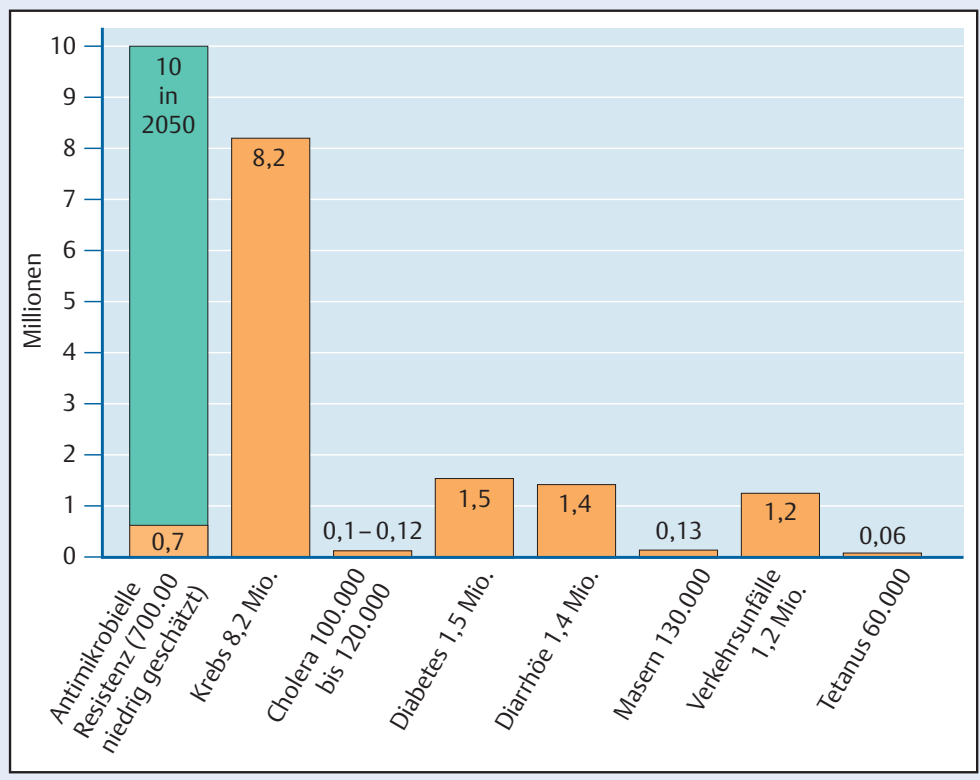

Abb. 1 Geschätzte Todesfälle jetzt und für das Jahr 2050 durch antimikrobielle Resistenz im Vergleich zu Todesfällen durch andere Erkrankungen [5]. se sind beunruhigend: Bis zum Jahr 2050 könnte die Zahl der Infektionen mit Krankheitserregern, gegen die keine Medikamente helfen, explodieren (aus dem Abschlussbericht Mai 2016: s. Abb. 1).

Unternehme man nichts gegen diese Ausbreitung, könnten ab dem Jahr 2050 zehn Millionen Menschen an Infektionen sterben, gegen die es keine Medikamente gibt. Der britische Schatzkanzler George Osborne formuliert auf einer Tagung in Washington: „Antimikrobielle Resistenz wird eine größere Bedrohung für die Menschheit werden als Krebs.“ Und als Diabetes. Und als Autounfälle. Und wahnsinnig teuer ist es natürlich auch: laut Studie würden die kumulativen wirtschaftlichen Kosten bei etwa 90 Billionen Euro liegen [3].

„Zehn Millionen Tote. Pro Jahr. Eine Zahl, die Angst macht. Die Angst machen soll“. [4]

Die Zahl - unkommentiert - ist nämlich falsch. Boytchev schreibt in seiner lesenswerten Analyse [4]: „Tatsächlich zeigt die Studie - und die Berichterstattung darüber - etwas Anderes: Dass sich Forscher und Medien inzwischen überbieten im Ausmalen von Schreckensszenarien, wenn es um Resistenzen geht. Dass sie Behauptungen machen, die auf lückenhaften Daten beruhen. Dass die Berichterstattung über Antibiotikaresistenzen immer schriller wird. In einem fort soll Aufmerksamkeit geschürt werden für ein vermeintlich unerkanntes Problem - das längst zu einem medialen Hype geworden ist.

Dahinter steckt auch Eigeninteresse. Forscher wollen höhere Etats, die Pharmaindustrie hofft auf Subventionen. Auch bei der britischen Studie waren die Konzerne beratend tätig. Eine der Schlussfolgerungen des Berichts lautet: Pro neuem Antibiotikum rund eine Milliarde Dollar an Zuschüssen zu vergeben.“

Also, wie kommen die Autoren der Studie auf solche unglaublichen Zahlen? 
Für ihre Berechnungen nahmen sie insgesamt sechs Mikroben/Erkrankungen (d.h. nicht nur Bakterien, sondern auch Viren und Parasiten):

- HIV

- Malaria

- Tuberkulose

- Klebsiella pneumoniae

- Escherichia coli und

- Staphylococcus aureus

Dann legen sie zwei Szenarien für ihre Hochrechnung zugrunde. Das erste Szenario geht davon aus, dass die Resistenzen für alle diese sechs Erreger um 40\% zunehmen. Außerdem rechneten die Autoren mit einer - völlig unplausiblen - Verdopplung der Ansteckungsrate. Das zweite Szenario ist noch unrealistischer, da es den Prinzipien der Biologie widerspricht: 100\% Resistenz [3]. D. h. kein einziges Antiinfektivum würde gegen Malaria und Tuberkulose, gegen HIV oder E. coli wirken. Der Großteil der Toten entfällt dann auf Tuberkulose und Malaria - Infektionskrankheiten, die die meist armen Menschen auf der Südhalbkugel bedrohen.

Im letzten Jahrhundert gab es einen dramatischen Rückgang bei Infektionskrankheiten insbesondere in den Industriestaaten. Dieser Rückgang war zwar auch auf Antiinfektiva zurückzuführen, aber ungleich viel mehr auf verbesserte sozioökonomische und hygienische Bedingungen und Impfungen. Warum soll das nicht auch für die Entwicklungs- und Schwellenländern gelten? Erfolg versprechen meines Erachtens vor allem bessere soziale Bedingungen und eine verbesserte Infektionsprävention und nicht eine Subventionierung der Pharmaindustrie, die mit sehr viel Geld motiviert werden möchte, nach neuen Antibiotika/Antiinfektiva zu forschen.
Ach ja - nach HIV und Malaria noch ein Wort zu multiresistenten Bakterien: Bei den Katastrophen-Exposés um ein postantibiotisches Zeitalter wird oft unterschlagen, dass resistente Erreger per se nicht mehr Infektionen als nicht-resistente Erreger verursachen. D. h. die Anzahl der Infektionen im ambulanten Bereich bliebe gleich (vorausgesetzt Immunkompetenz und Hygiene bleiben gleich). Ob eine bakterielle Infektion auftritt oder eben nicht, ist unabhängig vom Vorhandensein von Resistenzeigenschaften eines Erregers. Es hängt wesentlich davon ab, ob das körpereigene Immunsystem in der Lage ist, eine Infektion wirksam zu bekämpfen.

Um nicht missverstanden zu werden: Natürlich ist antimikrobielle Resistenz ein Problem. Die vielzitierten Daten der britischen Regierung übertreiben aber ziemlich und das Wort „ziemlich“ ist hier mit britischem understatement zu verstehen.

\section{Literatur}

1 Kittel J, Wagner J. Weltquartett. ; Stand: 26.07.2016 Im Internet: http://www.weltquartett.de/site/seuchen.html

2 Patalong F. Spiegel Online. Katastrophen-Quartette: Böses Spielchen. 07. 12 2011: Im Internet: http://www.spiegel.de/ panorama/katastrophen-quartette-boeses-spielchen-a801241.html ; Stand: 27.07.2016

3 O’Neill J. Tackling Drug-Resistant infections globally: final report and recommendations. Im Internet: http://amrreview.org/sites/default/files/160525_Final\%20paper_with\% 20cover.pdf (Stand: 26.07.2016)

4 Boytchev H. Ängste vor Bakterien. CORRECTIV - Recherchen für die Gesellschaft. Im Internet: https://correctiv.org/ recherchen/keime/artikel/2016/05/24/schrille-toene/ (Stand: 26.07.2016)

5 O'Neill J. Review on Antimicrobial Resistance. Tackling drugresistant infections globally. Im Internet: http://amr-review. org/file/111 (Stand: 26.07.2016) 